\title{
Traffic Aware Pilot De-contamination for Multi-Cell MIMO Systems
}

\author{
Praneeth Jayasinghe, Antti Tölli, Jarkko Kaleva, Ganesh Venkatraman, and Matti Latva-aho \\ Centre for Wireless Communications, University of Oulu, P.O. Box 4500, FIN-90014, Oulu, Finland. \\ \{praneeth.laddu, antti.tolli, jarkko.kaleva, ganesh.venkatraman, matti.latva-aho\}@oulu.fi
}

\begin{abstract}
Traffic aware precoder/decoder design in multi-cell multi-user multiple-input multiple-output systems is considered with the objective of weighted queue minimization, where the original non-convex optimization problem is solved via successive convex approximation. Centralized pilot reuse algorithms for mitigating the pilot contamination are investigated to reflect the traffic aware optimization objective. Distinctive feature of the proposed pilot reuse algorithms is to utilize the user buffer state information jointly with the traditional large scale fading values when allocating the limited pilot resources among the served users. Numerical examples compare the performance of the proposed pilot reuse algorithms for varying number of available pilots and different traffic arrival models. The results demonstrate that significant performance gains are available when the pilot allocation strategy is designed to reflect closely the overall system optimization objective.
\end{abstract}

\section{INTRODUCTION}

It is foretold that there is an enormous interest for mobile data traffic in the forthcoming years [1]. Small-cell deployment is identified as a promising way to satisfy this forever growing traffic in mobile data networks. This can be achieved with the use of a large number of small cells with very short coverage in addition to macro/micro cells, in high mobile data traffic areas [2]. At the same time, multiple-input multiple-output (MIMO) techniques can be employed to increase the spectral efficiency and improve the reliability of the mobile network [3]. Furthermore, MIMO systems can be used to mitigate the interference induced in multi-cell systems with the coordinated beamformer design [4]-[7].

Various studies on the coordinated beamforming design to optimize the network utilities such as weighted sum rate (WSR), energy efficiency and weighted queue were proposed for multi-cell multi-user MIMO systems. [4]-[9]. These studies consider designing beamformers in a centralized and decentralized manner with different coordination assumptions. Accurate estimation of channel state information (CSI) at the base station (BS) is essential for beamformer design in a multi-user multi-cell MIMO system. However, the number of orthogonal pilot sequences that can be used for channel estimation process is limited especially for a short coherence

This research was supported by Finnish Funding Agency for Technology and Innovation (TEKES), Nokia Networks. interval and/or a long channel delay spread [10], [11]. Hence, a large multi-cell MIMO network experiences the pilot contamination effect, due to interference from non-orthogonal pilots. Thus, it would be a limiting factor on the performance of multicell MIMO systems. The impact of pilot contamination and ways to mitigate this effect have been studied for multicell MIMO systems [12]-[15]. Blind pilot decontamination is considered in [12]. They proposed a channel predicting approach by utilizing the properties of random matrix theory. In [13], spatial correlation properties are utilized for reusing the pilot resources among users with sufficient angular separation. Achievable rates in a contaminated multiuser MIMO system is analyzed for massive MIMO [14] and for precoding based on MMSE [15] is analyzed by using of large scale fading.

Motivated by above concerns, we consider precoder/decoder design to minimize total backlogged packets of a multicell multi-user MIMO system with the availability of limited pilot resources. The weighted queue minimization approach from [9] is used as the framework to solve the beamformer optimization problem. Moreover, centralized pilot reuse algorithms are investigated for mitigating the pilot contamination to reflect the traffic aware optimization objective of the beamformer design. Proposed pilot reuse algorithms are utilizing details of traditional large-scale fading and the user buffers. Then, we proposed a channel estimation method to extract the individual channel from the contaminated channels by using the large-scale fading information. Proposed decontamination methods numerically investigated in comparison to perfect CSI case and random pilot allocation scheme.

The rest of the paper is organized as follows. Section II explains the system model and in Section III, we formulate the weighted queue minimization problem. Section IV presents the proposed pilot decontamination methods and Section $\mathrm{V}$ presents the numerical examples. Section VI concludes the paper.

\section{SySTEM MODEL}

We consider a multi-cell multi-user MIMO system operating in time division duplexing (TDD) mode. The multi-cell network consists of $N$ BSs and $K$ users. Each BS $i$ and user $k$ is equipped with $N_{T}$ and $N_{R}$ antennas, respectively. We denote the set of $\mathrm{BS}$ indices as $\mathcal{B}$, the set of users that are served by BS $i$ is denoted as $\mathcal{U}_{i}$ and set $\mathcal{U}$ represents all users 
in the system. The number of users in BS $i$ is $\left|\mathcal{U}_{i}\right|$. Data for user $\mathrm{k}$ is transmitted from only one BS which is denoted by $b_{k} \in \mathcal{B}$. Furthermore, we assume the channel reciprocity of the wireless channel to obtain the CSI.

The estimated received data $x_{l, k}$ corresponding to $l$ th spatial stream at the down-link (DL) user $k$ can be expressed as,

$$
\begin{aligned}
x_{l, k}= & \mathbf{w}_{l, k}^{\mathrm{H}} \mathbf{H}_{b_{k}, k} \mathbf{m}_{l, k} d_{l, k}+\mathbf{w}_{l, k}^{\mathrm{H}} \sum_{i \in \mathcal{U} \backslash\{k\}} \mathbf{H}_{b_{i}, k} \sum_{j=1}^{L} \mathbf{m}_{j, i} d_{j, i} \\
& +\mathbf{w}_{l, k}^{\mathrm{H}} \mathbf{n}_{l, k}
\end{aligned}
$$

where $\mathbf{m}_{l, k} \in \mathbb{C}^{N_{T} \times 1}$ is the transmit beamformer at the DL BS $b$ for user $k$ on the $l$ th spatial stream, $\mathbf{w}_{l, k} \in \mathbb{C}^{N_{R} \times 1}$ is the receive beamforming vector at the DL user $k$. The BS-User channel is denoted as $\mathbf{H}_{b, k} \in \mathbb{C}^{N_{R} \times N_{T}}$. Additive noise vector for user $k$ on the $l$ spatial stream is $\mathbf{n}_{l, k} \sim \mathcal{C N}\left(0, N_{0}\right)$. The data symbol for DL user $k$ on the $l$ th spatial stream is denoted as $d_{l, k}$ and $L=\min \left(N_{T}, N_{R}\right)$ is the maximum number of spatial streams.

Assuming the independent detection of data streams, we can write the signal-to-interference-plus-noise ratio (SINR) at the DL user $k$ as,

$$
\gamma_{l, k}=\frac{\left|\mathbf{w}_{l, k}^{\mathrm{H}} \mathbf{H}_{b_{k}, k} \mathbf{m}_{l, k}\right|^{2}}{\hat{N}_{0}+\sum_{i \in \mathcal{U} \backslash\{k\}} \sum_{j=1}^{L}\left|\mathbf{w}_{l, k}^{\mathrm{H}} \mathbf{H}_{b_{i}, k} \mathbf{m}_{j, i}\right|^{2}}
$$

where $\hat{N}_{0}=N_{0}\left\|\mathbf{w}_{l, k}\right\|^{2}$ denotes the equivalent noise variance.

Let $Q_{k}$ be the number of queued packets at a given scheduling instant for DL user. The total number of queued packets at the $(i+1)$ th time instant at DL BS destined to user $k$ is given by

$$
Q_{k}(i+1)=\left[Q_{k}(i)-t_{k}(i)\right]^{+}+\lambda_{k}(i)
$$

where $[x]^{+} \equiv \max \{x, 0\}, t_{k}$ is the transmission rate to DL user. Instantaneous traffic generated at the time instant $i$ for DL user is denoted $\lambda_{k}(i)$. Also, for SINR $\gamma_{l, k}$, the maximum rate achieved is upperbounded by $t_{l, k} \leq \log _{2}\left(1+\gamma_{l, k}\right)$.

\section{PROBLEM FORMULATION}

We consider weighted $\ell_{q}$-norm queue minimization for the downlink transmission considering the queues destined to DL users with power constraints on the DL BSs. In this section, we follow the same optimization frame work used in [9]. However, for the sake of clarity, we summarize the basic steps. The queue deviation metric is defined as

$$
\Psi_{k}=Q_{k}-t_{k}=Q_{k}-\sum_{l=1}^{L} \log _{2}\left(1+\gamma_{l, k}\right) .
$$

Then, define the optimization objective as $\sum_{k \in \mathcal{U}} a_{k}\left|\Psi_{k}\right|^{q}$, where $a_{k} \forall k$ are the weighting factors used to prioritize users based on the quality of service (QoS) constraints. Note that the objective includes an implicit rate constraint $Q_{k}-\sum_{l=1}^{L} t_{l, k} \geq$ 0 . Then, the optimization problem can be formulated by relaxing the SINR expressions as inequality constraints and introducing new variables $\gamma_{l, k}$, and $\beta_{l, k}$ as

$$
\begin{array}{cc}
\underset{\substack{\mathbf{m}_{l, k}, \mathbf{w}_{l, k} \\
\gamma_{l, k}, \beta_{l, k}}}{\operatorname{minimize}} & \|\tilde{\boldsymbol{\Psi}}\|_{q} \\
\text { subject to } & \gamma_{l, k} \leq \frac{\left|\mathbf{w}_{l, k}^{\mathrm{H}} \mathbf{H}_{b_{k}, k} \mathbf{m}_{l, k}\right|^{2}}{\beta_{l, k}} \\
& \beta_{l, k} \geq N_{0}+\sum_{i \in \mathcal{U} \backslash\{k\}} \sum_{j=1}^{L}\left|\mathbf{w}_{l, k}^{\mathrm{H}} \mathbf{H}_{b_{i}, k} \mathbf{m}_{j, i}\right|^{2} \\
& \sum_{k \in \mathcal{U}_{b}} \sum_{l=1}^{L}\left\|\mathbf{m}_{l, k}\right\|^{2} \leq P_{\max } \quad \forall b
\end{array}
$$

where $\tilde{\Psi}_{k} \triangleq a_{k}^{1 / q} \Psi_{k}$ are the elements of the vector $\tilde{\boldsymbol{\Psi}}$. The BS specific power constraint is considered in $(5 \mathrm{~d})$. The $\ell_{q}$ norm is used to provide the tradeoff between fairness and sum queue minimization.

This is a non-convex optimization problem with the optimization variables of $\mathbf{m}_{l, k}, \mathbf{w}_{l, k}, \gamma_{l, k}, \beta_{l, k}(\forall l, k)$. Note that (5d) is the only convex constraint with the involved variables. For fixed receivers $\mathbf{w}_{l, k},(5 \mathrm{c})$ can be written as a second-order cone (SOC) constraint. Now, by using the first order Taylor approximation around the operating point, we can approximate (5b) as [9], [16]-[19]

$$
\begin{aligned}
\gamma_{l, k} \leq 2 \frac{\tilde{p}_{l, k}}{\tilde{\beta}_{l, k}}\left(p_{l, k}-\tilde{p}_{l, k}\right)+2 \frac{\tilde{q}_{l, k}}{\tilde{\beta}_{l, k}}\left(q_{l, k}-\tilde{q}_{l, k}\right) \\
+\frac{\tilde{p}_{l, k}^{2}+\tilde{q}_{l, k}^{2}}{\tilde{\beta}_{l, k}}\left(1-\frac{\beta_{l, k}-\tilde{\beta}_{l, k}}{\tilde{\beta}_{l, k}}\right)
\end{aligned}
$$

where define $\psi_{l, k} \triangleq\left\{\mathbf{w}_{l, k}, \mathbf{m}_{l, k}, \beta_{l, k}\right\}$,

$$
g\left(\psi_{l, k}\right) \triangleq \frac{\left|\mathbf{w}_{l, k}^{\mathrm{H}} \mathbf{H}_{b_{k}, k} \mathbf{m}_{l, k}\right|^{2}}{\beta_{l, k}}
$$

and $p_{l, k} \triangleq \Re\left(\mathbf{w}_{l, k}^{\mathrm{H}} \mathbf{H}_{b_{k}, k} \mathbf{m}_{l, k}\right), q_{l, k} \triangleq \Im\left(\mathbf{w}_{l, k}^{\mathrm{H}} \mathbf{H}_{b_{k}, k} \mathbf{m}_{l, k}\right)$ Hence, $g\left(\psi_{l, k}\right)=\left(p_{l, k}^{2}+q_{l, k}^{2}\right) / \beta_{l, k}$. Let $\tilde{\psi}_{l, k} \triangleq$ $\left\{\tilde{\mathbf{w}}_{l, k}, \tilde{\mathbf{m}}_{l, k}, \tilde{\beta}_{l, k}\right\}$ be a minimizer from the previous SCA iteration. In summary, for fixed receivers $\tilde{\mathbf{w}}_{l, k}$ and operating point $\tilde{\psi}_{l, k}$ the relaxed convex subproblem for finding transmit precoders is given by

$$
\begin{array}{ll}
\underset{\substack{\mathbf{m}_{l, k}, \gamma_{l, k}, \beta_{l, k} \\
\text { subject to }}}{\operatorname{minimize}} \quad(6) \text { and (5d) } \|_{q} \\
\qquad \beta_{l, k} \geq \hat{N}_{0}+\sum_{\substack{b \in \mathcal{B} \\
i \neq k}} \sum_{j=1}^{L}\left|\tilde{\mathbf{w}}_{l, k}^{\mathrm{H}} \mathbf{H}_{b_{i}, k} \mathbf{m}_{j, i}\right|^{2}
\end{array}
$$

Now, the optimal receivers for fixed transmit precoders $\tilde{\mathbf{m}}_{l, k}$ are obtained by minimizing (5) w.r.t. $\mathbf{w}_{l, k}$ as 


$$
\begin{array}{ll}
\underset{\substack{\mathbf{w}_{l, k}, \gamma_{l, k}, \beta_{l, k} \\
\text { subject to }}}{\operatorname{minimize}} & \|\tilde{\boldsymbol{\Psi}}\|_{q} \\
& \beta_{l, k} \geq \hat{N}_{0}+\sum_{\substack{b \in \mathcal{B} \\
i \neq k}} \sum_{j=1}^{L}\left|\mathbf{w}_{l, k}^{\mathrm{H}} \mathbf{H}_{b_{i}, k} \tilde{\mathbf{m}}_{j, i}\right|^{2}
\end{array}
$$

Then, repeat the steps in (8) and (9) until the objective is converged [9].

\section{Pilot Decontamination}

The proposed precoder/decoder beamformer algorithm is a centralized design assuming TDD mode. Thus, in order to solve the above optimization problem (5), all the BSuser channels should be perfectly known to the centralized unit. As per the TDD mode assumption, we employ uplink training to estimate CSI at the BS with the use of channel reciprocity. Then we assume that BSs inform all the user channels to the centralized unit. The centralized unit estimates the transmit precoders with the available information. Then, it distributes the transmit precoders to the BSs. However, in a large multiuser multi-cell MIMO environment, we may run out of orthogonal pilot sequences which are available for uplink training. This is mainly due to the limitation of coherence bandwidth and coherence time of the wireless communication channel [10], [11]. Thus, we have to share the same pilot resource with other users in nearby cells for the channel estimation process. As a result, pilot interference from neighboring cells would limit the accuracy of the estimated channel. Therefore, the channel estimates obtained in the cell of interest are contaminated by signals transmitted by users in the other cells. This is called the pilot contamination.

The effect of pilot contamination and its decontamination have been studied in numerous papers in the context of multicell MIMO system [12]-[15]. However, most of these studies were focussed on investigating this effect for massive MIMO systems. Hence, they proposed the solutions using statistical properties of random matrix theory and often assuming simple match filter/channel hardening. Moreover, most of these studies considered repetitive pilot assignments over the cells. This is a very inefficient assumption to study the performance because the number of users scheduled in a given time instant may differ one cell to another. Additionally, according to the author's knowledge, there have been very limited studies on pilot decontamination while optimizing network utility such as weighted queue minimization. Hence, it is important to study the above precoder/decoder design (5), in a limited pilot resources environment. Pilot contamination effect of the system can be demoted in several ways, such as allocating pilots to minimize the pilot overlap and using clever channel estimation algorithms. In this section, we discuss the ways to mitigate this pilot contamination in such a way that it supports the original optimization objective. Here, we suggest 3 types of pilot decontamination methods, named Logarithmically weighted interference to signal ratio (ISR) based decontamination, Queue based decontamination and Hybrid decontamination.

\section{A. Logarithmically weighted ISR based decontamination}

One way to mitigate pilot contamination from the system is the use of intelligent pilot allocation, that minimizes the interference of the contaminated channel. Here, we are presenting a novel pilot allocation algorithm by using path loss details.

When two users share the same pilot sequence, both BSUser channels are corrupted by each other. Assuming both users have the same transmit powers, the distance between interfering users is considered as a dominant factor when assigning pilots to each other. We can get a rough idea about the distance between interfering users using long-term statistics (i.e. path loss details) of the BS-user channels. Here, we define the utility function $R_{\mathcal{P}_{i}}$ for pilot $i \in\{1, \ldots, N\}$ that shared with set of users $\mathcal{P}_{i} \subset \mathcal{U}$ as,

$$
R_{\mathcal{P}_{i}}=\sum_{k \in \mathcal{P}_{i}} \log \left(1+\sum_{j \in \mathcal{P}_{i} /\{k\}} I_{j} / S_{k}\right)
$$

where $S_{k}$ is the path gain between the user $k$ and its serving BS $b_{k}, I_{j}$ is the path gain between the user $j$ and adjacent BS $b_{k}$. Here, we use this logarithmic ISR term to provide fairness among users (otherwise strong user could dominate among all $\mathcal{P}_{i}$ ). Also, $1+$ term is to make the utility function always positive. Then the unconstrained minimization problem which is used for the pilot allocation is given by,

$$
\underset{\mathcal{P}_{i} \forall i}{\operatorname{minimize}} \sum_{i=1}^{N} R_{\mathcal{P}_{i}}
$$

Note that the objective (11) is minimizing total rate loss induced due to pilot contamination. However, the correlation between beamformer design and the pilot allocation algorithm is less as the beamformer design utilizes user traffic information for optimization algorithm and pilot allocation algorithm does not. For example, two users may assign into the same pilot with larger queues. Those users may minimize (11) while destroying the objective of (5).

\section{B. Quеиe based decontamination}

Since the performance of the earlier method is constrained without user buffer details, we propose user buffer based decontamination method. In order to avoid the earlier scenario, users with less packets in the buffer are grouped into same pilot. Here, we define utility function $W_{\mathcal{P}_{i}}$ as,

$$
W_{\mathcal{P}_{i}}=\sum_{k \in \mathcal{P}_{i}} Q_{k}
$$

where $Q_{k}$ is the queued packets at the user $k$. Then the pilot allocation based on the unconstrained minimization problem is given by,

$$
\underset{\mathcal{P}_{i} \forall i}{\operatorname{minimize}} \sum_{i=1}^{N} W_{\mathcal{P}_{i}} .
$$

By grouping users with lower buffer, we are trying to lower the interference to the users with larger queues. However, in this 
method two nearby users may use the same pilot which may negatively affect to the objective in (5). Note that user buffers are used only for the pilot allocation algorithm while path loss details will be used to estimate the channels as in the Section IV-D. Thus, this is more like a pilot decontamination method which uses both user buffer details and path loss details.

\section{Hybrid decontamination}

Both pilot allocation algorithms were discussed above have their own limitations. Even though they are trying to allocate pilots in the best possible way, it may not be matched with the objective of the original precoder/decoder design. Therefore, we propose a pilot allocation algorithm which utilizes both user buffer and path loss details for pilot allocation. Here, we define the utility function $G_{\mathcal{P}_{i}}$ as,

$$
G_{\mathcal{P}_{i}}=\sum_{k \in \mathcal{P}_{i}} Q_{k} \log \left(1+\sum_{j \in \mathcal{P}_{i} /\{k\}} I_{j} / S_{k}\right)
$$

Here, logarithmic ISR term is used for the same reason mentioned in Section IV-A, to provide fairness among users. Then the unconstrained minimization problem used for the pilot allocation can be formulated as,

$$
\underset{\mathcal{P}_{i} \forall i}{\operatorname{minimize}} \sum_{i=1}^{N} G_{\mathcal{P}_{i}}
$$

Since the Hybrid method uses both user buffer and path loss information, highly interfering users will not be assigned to users with larger buffers. Therefore, this approach is quite supportive to the objective in original optimization problem (5).

Above decontamination algorithms in (11), (13) and (15) need exhaustive search to obtain the optimal pilot allocation. However, as the number of users in the network increases, it requires lots of computational time. Thus, we use a simplified algorithm which provides quite similar results. Proposed decontamination methods are summarized as in Algorithm 1.

\section{Channel estimation}

In a contaminated channel, desired user channel is corrupted by the other user channels due to several users are sharing the same pilot for uplink training. However, we need to estimate each BS-user channel (desired BS-user and interference channels) individually to apply for the optimization framework in (5). We can model the contaminated channel which uses the pilot resource $i$ as,

$$
\mathbf{H}^{i}=\sum_{j \in \mathcal{P}_{i}} \mathbf{H}_{j}^{i}+\mathbf{N}
$$

where $\mathbf{H}^{i}$ is the contaminated channel that used the pilot resource $i$ and $\mathbf{H}_{j}^{i}$ is the BS-user $j$ channel. In order to separately estimate each BS-user channels, we require the pilot reuse information and BS-user path loss details. Hence we can estimate the desired user channel $\hat{\mathbf{H}}_{k}^{i}$ as [20]

$$
\hat{\mathbf{H}}_{k}^{i}=\frac{\sqrt{g_{k}}}{\sum_{j \in \mathcal{P}_{i}} \sqrt{g_{j}}} \mathbf{H}^{i}
$$

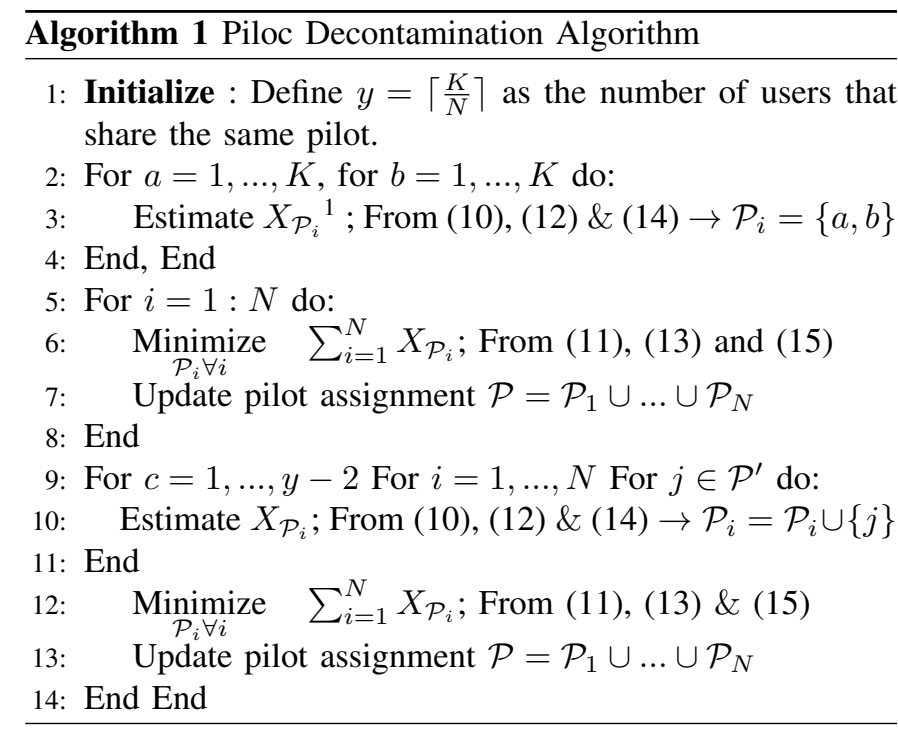

where $g_{j}$ are the path loss information of the user $j$.

\section{NUMERICAL EXAMPLES}

Performance of pilot reuse methods is investigated by changing simulation parameters such as traffic arrival rate and number of available pilot resources. In the simulation model, we consider 3 sectored 7 cell wrap-up model with 2 users in each sector. The number of antennas at each BS is $N_{T}=4$ and the number of antennas at each user terminal is $N_{R}=2$. The simulation environment is modeled as the [21]. Poisson arrival process is used to model the traffic generation in a given time instance at the DL BSs for user $k, \lambda_{k}(i) \sim \operatorname{Pois}\left(A_{k}\right)$ with the average number of traffic arrivals of $A_{k}=\mathbf{E}_{i}\left\{\lambda_{k}^{D}\right\}$ packets or bits. Also, pilots within the same cell are considered to be orthogonal and $20 \%$ of users considered to be idle (empty buffers). All the simulations are obtained with 50 iterations per user drop.

Fig. 1 presents the comparison of each pilot reuse method when the available number of pilots is equal to 15 . The average arrival rate $\left(\sum_{k} A_{k} / K\right.$ ) over all users is considered in the $\mathrm{x}$-axis. Asymmetric traffic is generated with 3 user groups such as user group 1 consisting of one-third of the users with average arrival rate $0.5 A_{k}$, user group 2 consisting of half of the users with average arrival rate $A_{k}$ and user group 3 consisting of one-sixth of the users with average arrival rate $2 A_{k}$. Several user groups are considered to observe the impact of traffic variation for each pilot decontamination method. Perfect CSI case (orthogonal pilots case which requires 42 pilots to estimate all the channels perfectly) and Random pilot allocations (randomly assigned pilots to each user and uses the approach presented in Section IV-D for the channel estimation) are used as the reference scenarios. Apart from the Random pilot allocation, the Queue based case provides the worst pilot decontaminated performance. This is due to highly interfering users are allocating to the same pilot resource. The

$$
{ }^{1} X_{\mathcal{P}_{i}} \text { is for } R_{\mathcal{P}_{i}}, W_{\mathcal{P}_{i}} \text { or } G_{\mathcal{P}_{i}}
$$




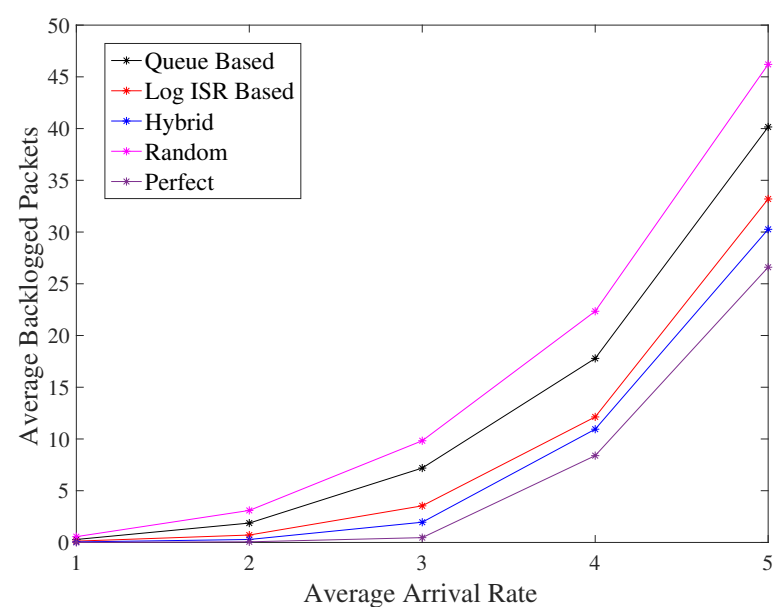

Fig. 1: Asymmetrical traffic distribution with $20 \%$ idle users and available orthogonal pilots is 15 and Cell Separation $=200 \mathrm{~m}$.

Path loss based method and the Hybrid method provide considerably better performance. The Logarithmically weighted ISR based method works well here due to this algorithm finds the user groups which have minimum interference to each other. Which indeed help to find individual channels with the very small error from (17). Thus the overall effect to precoder/decoder design would be minimal. However, in a heavy interference environment, users with high buffers may assign to the same pilot resource. That can be affected badly to the overall system throughput. By using both path loss and buffer states, the Hybrid method provides better performance than other approaches. This is because of the objectives of the precoder/decoder design and pilot reuse utility function are correlated.

Fig. 2 illustrates the performance of total backlogged packets of each pilot reuse method vs the number of orthogonal pilot resources. An asymmetrical traffic model is considered as in the earlier case at the average traffic arrival rate of 5. In the Hybrid method, in comparison to perfect case the throughput loss is lesser than 5 packets/bit when the number of pilots is 12 . That is for utilizing a $71 \%$ lesser consumption of pilot resources. Thus, Hybrid scheme significantly improves the effectiveness of the pilot resources in comparison to the orthogonal scenario. In The Logarithmically weighted ISR based method, rate loss is around 8 packets/bits for the same number of pilots. Moreover, it performs much better as compared to Queue based method and Random method. However, all the pilot decontamination methods start to degrade their effectiveness when the number of orthogonal pilots is less than 12. Fig. 3 illustrates the performance of each pilot reuse method as the earlier case by considering symmetric traffic with the average traffic arrival rate $A_{k}$ for all users is equal to 5 . By using symmetric traffic, we limit the traffic variation between users. However, the performance gain of the each method is quite similar to the earlier case.

Finally, Fig. 4 illustrates the performance of each pilot reuse method for a different number of pilot resources with

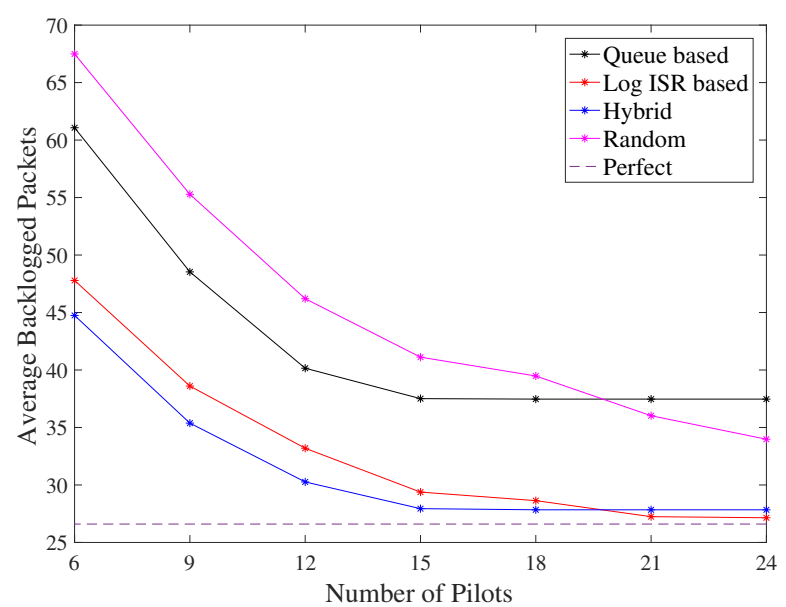

Fig. 2: Asymmetrical traffic distribution with overall average arrival rate of 5 and $20 \%$ idle users and Cell Separation $=200 \mathrm{~m}$.

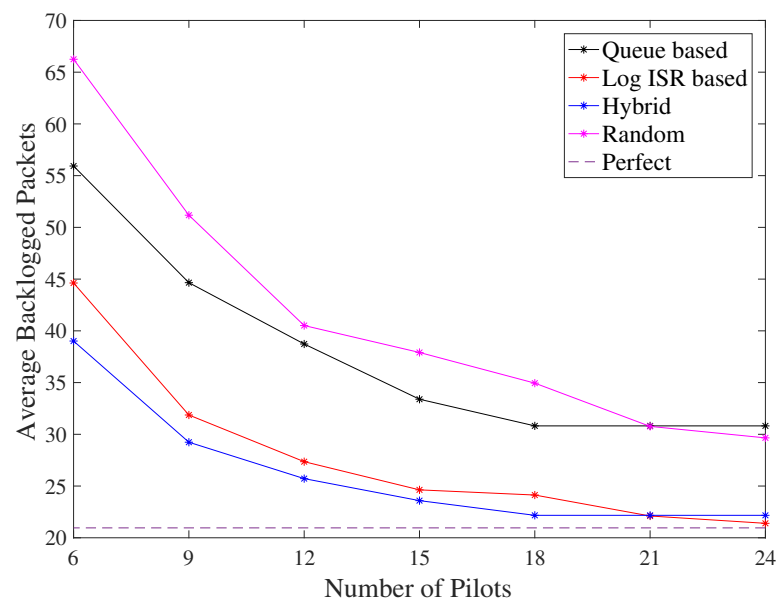

Fig. 3: Symmetrical traffic distribution with overall average arrival rate of 5 and $20 \%$ idle users and Cell Separation $=200 \mathrm{~m}$.

an asymmetrical traffic as in earlier cases (Fig.1 and Fig. 2) at average traffic arrival rate of 5 . However, we decrease the cell separation to $100 \mathrm{~m}$ (Cell separation is $200 \mathrm{~m}$ in Fig.1-3) in order to increase the interference in the channel estimation process. As the cells get closer, interfering BS-User distance are getting closer. Assuming the transmit power are same during UL channel training, this led to increasing the total interference at the network during channel estimation process. As expected, the Logarithmically weighted ISR based method performs badly as compared to Queue based method in heavy pilot reuse cases (more than 2 users share the same pilot). The reason could be assigning good pilots (overall channel estimation error is low) to zero traffic users and bad pilots (overall channel estimation error is high) to users with large buffers. The Hybrid method performs pretty well even for the number of pilots are less than 12 .

\section{CONCLUSIONS}

In this paper, we study the pilot decontamination for traffic aware multicell MIMO system. Transmit precoders at the 


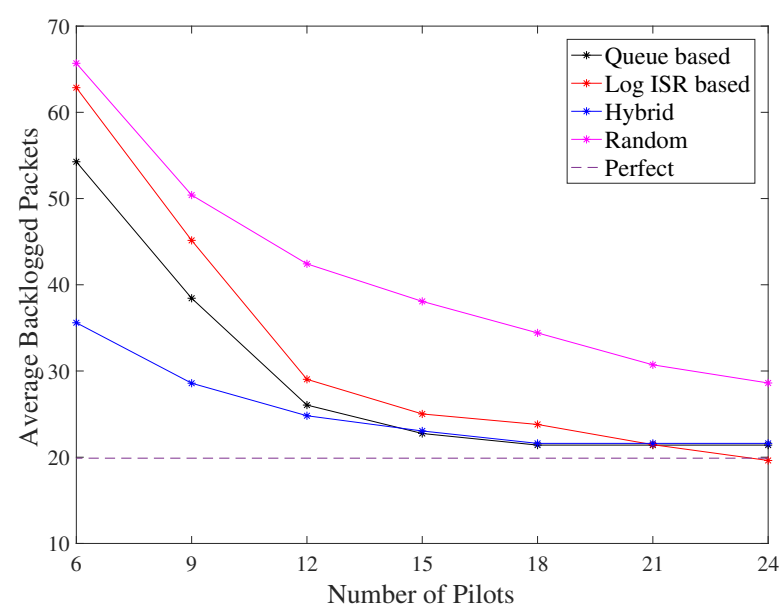

Fig. 4: Asymmetrical traffic distribution with overall average arrival rate of 5 and $20 \%$ idle users and Cell Separation $=100 \mathrm{~m}$.

BSs and combining receivers at the users are employed with the objective of weighted queue minimization. The original non-convex optimization problem is solved using successive convex approximation framework. In order to demote pilot contamination effect, several pilot reuse algorithms are proposed by using traffic and path loss details. Numerical results suggested that Hybrid method significantly improves the performance when compared to other methods. Pilot decontamination is improved when the correlation between the objectives of the beamformer design and the pilot reuse algorithm is higher. Having both traffic and path loss information is beneficial to mitigate the pilot contamination effect.

\section{REFERENCES}

[1] Cisco Systems, "Cisco Visual Networking Index: Global Mobile Data Traffic Forecast Update, 2015-2020," [Online Oct. 2016], http://www.cisco.com/c/en/us/solutions/collateral/service-provider/ visual-networking-index-vni/mobile-white-paper-c11-520862.html

[2] T. Nakamura, S. Nagata, A. Benjebbour, Y. Kishiyama, T. Hai, S. Xiaodong, Y. Ning, and L. Nan, "Trends in small cell enhancements in LTE advanced," IEEE Commun. Mag., vol. 51, no. 2, pp. 98-105, Feb. 2013.

[3] D. Tse and P. Viswanath, Fundamentals of wireless communication, Cambridge university press, 2005.

[4] Q. Shi, M. Razaviyayn, Z. Luo, and C. He, "An iteratively weighted MMSE approach to distributed sum-utility maximization for a MIMO interfering broadcast channel," IEEE Trans. Signal Processing, vol. 59, no. 9, pp. 4331-4340, Sept. 2011.

[5] P. Komulainen, A. Tolli, and M. Juntti, "Effective CSI signaling and decentralized beam coordination in TDD multi-cell MIMO systems," IEEE Trans. Signal Processing, vol. 61, no. 9, pp. 2204-2218, May. 2013.

[6] L. Venturino, N. Prasad, and X. Wang, "Coordinated linear beamforming in downlink multi-cell wireless networks," IEEE Trans. Wireless Commun., vol. 9, no. 4, pp. 1451-1461, Apr. 2010.

[7] K. Wang, X. Wang, W. Xu, and X. Zhang, "Coordinated linear precoding in downlink multicell MIMO-OFDMA networks," IEEE Trans. Signal Processing, vol. 60, no. 8, pp. 4264-4277, Aug. 2012.

[8] S. S. Christensen, R. Agarwal, E. Carvalho, and J. M. Cioffi, "Weighted sum-rate maximization using weighted MMSE for MIMO-BC beamforming design," IEEE Trans. Wireless Commun., vol. 7, no. 12, pp. 4792-4799, Dec. 2008.

[9] G. Venkatraman, A. Tölli, M. Juntti, and L. Tran, "Traffic Aware Resource Allocation Schemes for Multi-Cell MIMO-OFDM Systems,"
IEEE Trans. Signal Processing, vol. 64, no. 11, pp. 2730-2745, Jun. 2016.

[10] T. L. Marzetta, "Noncooperative cellular wireless with unlimited numbers of base station antennas," IEEE Trans. Wireless Commun., vol. 9, no. 11 , pp. 3590-3600, Oct. 2010.

[11] E. G. Larsson, O. Edfors, F. Tufvesson, and T. L. Marzetta, "Massive mimo for next generation wireless systems," IEEE Commun. Mag., vol. 52, no. 2, pp. 186-195, Feb. 2014.

[12] R. R. Müller, L. Cottatellucci, and M. Vehkaperä, "Blind pilot decontamination," IEEE J. Select Topics Signal Processing, vol. 8, no. 5, pp. 773-786, Mar. 2014.

[13] H. Yin, D. Gesbert, M. C. Filippou, and Y. Liu, "Decontaminating pilots in massive mimo systems," in IEEE Int. Conf. Commun. (ICC). IEEE, 2013, pp. 3170-3175.

[14] H. Q. Ngo, T. L. Marzetta, and E. G. Larsson, "Analysis of the pilot contamination effect in very large multicell multiuser MIMO systems for physical channel models," in IEEE Int. Conf. Acoustics, Speech and Signal Processing (ICASSP), 2011, pp. 3464-3467.

[15] J. Jose, A. Ashikhmin, T. L. Marzetta, and S. Vishwanath, "Pilot contamination and precoding in multi-cell TDD systems," IEEE Trans. Wireless Commun., vol. 10, no. 8, pp. 2640-2651, Aug. 2011.

[16] B. R. Marks and G. P. Wright, "Technical note?a general inner approximation algorithm for nonconvex mathematical programs," Operations Research, vol. 26, no. 4, pp. 681-683, 1978.

[17] G. R. Lanckriet and B. K Sriperumbudur, "On the convergence of the concave-convex procedure," in Advances in neural information processing systems, 2009, pp. 1759-1767.

[18] G. Scutari, F. Facchinei, L. Lampariello, and Peiran Song, "Parallel and distributed methods for nonconvex optimization- part i: Theory," IEEE Trans. Signal Process, 2014.

[19] S. Boyd and L. Vandenberghe, Convex optimization, Cambridge university press, 2004.

[20] T. Kailath, A. H. Sayed, and B. Hassibi, Linear estimation, vol. 1, Prentice Hall Upper Saddle River, NJ, 2000.

[21] 3GPP, "Technical report 36.814: Further advancements for E-UTRA physical layer aspects," Tech. Rep, 3rd Generation Partnership Project 3GPP, www.3gpp.org, 2010-03. 